\title{
Nuclear masses and the origin of the elements
}

\author{
Hendrik Schatz ${ }^{1}$, Klaus Blaum ${ }^{2}$ \\ 'Johannes Gutenberg-University Mainz and GSI Darmstadt • Germany. \\ ${ }^{2}$ National Superconducting Cyclotron Laboratory, Michigan State University • USA.
}

T he chemical composition of our universe has many surprising features: why does the sun consist of mainly hydrogen and helium? Why is iron so much more abundant than heavier elements such as gold? Why are there heavy elements at all and how did they come into existence? The properties of atomic nuclei, especially their masses, play a crucial role in these fundamental questions at the interface of nuclear and astrophysics.

Fig. 1 shows the distribution of the solar system isotopic abundances determined from the analysis of meteorites and the spectrum of sunlight. Many of the general features of the distribution of the solar system elemental abundances shown in Fig. 1 can be found throughout our Galaxy and probably the universe as a whole, though there are variations owing to differences in nucleosynthetic history or ongoing nucleosynthesis processes. Hydrogen and helium are by far the most abundant elements in the solar system. These elements were already produced in the big bang, together with traces of lithium. All the heavier elements must have been created after the big bang by nuclear processes, mostly in stars and stellar explosions. A look at the nuclear masses explains why the big bang was not able to produce heavy elements using the already formed hydrogen $\left({ }^{1} \mathrm{H}\right)$ and helium $\left({ }^{4} \mathrm{He}\right)$. Among the possible nuclear fusion reactions one could imagine are the fusion of two hydrogen nuclei into ${ }^{2} \mathrm{He}$, the fusion of a hydrogen nucleus with a helium nucleus producing ${ }^{5} \mathrm{Li}$, or the fusion of two helium nuclei into ${ }^{8} \mathrm{Be} .{ }^{2} \mathrm{He},{ }^{5} \mathrm{Li}$, and ${ }^{8} \mathrm{Be}$ have one thing in common: in contrast to the isotopes usually found on earth and in stars these nuclei have extremely short lifespans - for example, in the case of ${ }^{8} \mathrm{Be}$ the average lifetime is just $10^{-16}$ seconds. The reason is that the decay of these nuclei into fragments is energetically possible because the total mass of the fragments is smaller than the mass of the nucleus itself. This mass difference corresponds according to Einstein's famous equation $E=m c^{2}$ to the binding energy that can be released during the decay. ${ }^{2} \mathrm{He},{ }^{5} \mathrm{Li}$, and ${ }^{8} \mathrm{Be}$ are destroyed by

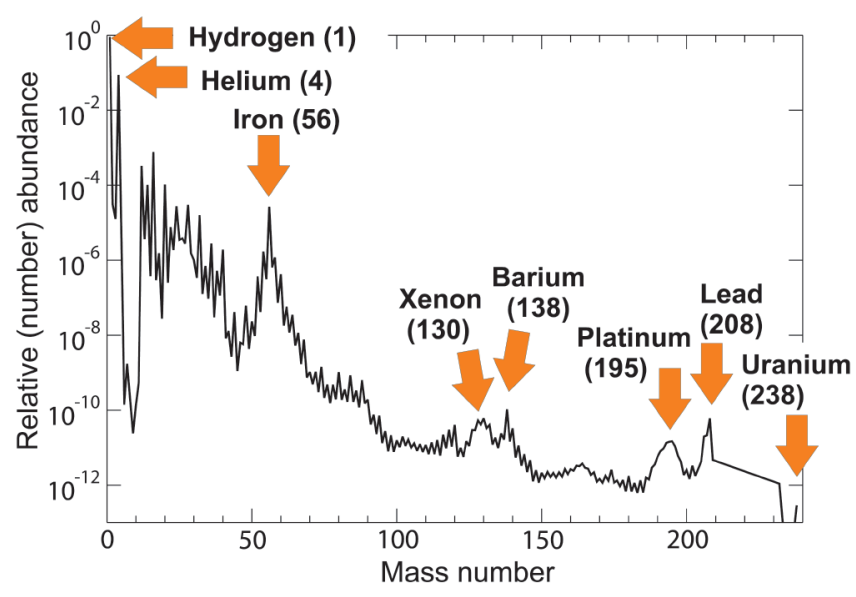

$\Delta$ Fig. 1: The relative abundance of the isotopes of the chemical elements in the solar system [1] as a function of their mass number (the total number of protons and neutrons in the nucleus). Some of the abundance peaks are marked with the mass number and the element that dominates the composition at this point. decay immediately after they are formed and can therefore, on average, only exist in extremely small quantities. The densities and the amount of time available for nuclear reactions in the big bang are not sufficient to initiate any further reactions on these small amounts of ${ }^{2} \mathrm{He},{ }^{5} \mathrm{Li}$, and ${ }^{8} \mathrm{Be}$. If the mass of the ${ }^{8} \mathrm{Be}$ nucleus would be smaller by just about a 1/1000 of a percent, the decay into two helium nuclei could not occur anymore and ${ }^{8}$ Be would be stable. In this case, heavy elements could have been produced easily during the big bang, with drastic consequences for the existence of stars, including the sun, which uses the fusion of hydrogen from the big bang into heavier elements as its main energy source.

Another striking feature in Fig. 1 is the relatively large abundance of nuclei around iron and nickel. This can again be explained by looking at the nuclear masses. The difference between the actual nuclear mass and the total mass of the nucleons ( $Z$ protons and $N$ neutrons) determines through $E=m c^{2}$ the binding energy of the protons and neutrons in the nucleus. As an example, in ${ }^{4} \mathrm{He}$ this binding energy amounts to about $1 \%$ of the total mass. It turns out, that the nuclei around iron and nickel have the largest binding energies per nucleon of all stable nuclei. For example, a nucleon in ${ }^{56} \mathrm{Fe}$ is bound on average by $8.79 \mathrm{MeV}=1.41 \cdot 10^{-12} \mathrm{~J}$ [2]. Because of this, neither the fusion of two iron nuclei nor fission of an iron nucleus into smaller constituents releases energy - in fact one would need to provide energy to make these processes happen. As a consequence, the chain of nuclear fusion reactions in the interior of stars that produces energy by converting nuclei with less binding energy into nuclei with more binding energy per nucleon ends at iron and nickel. Earlier generations of stars have already begun to burn a small part of the hydrogen and helium from the big bang into iron and nickel. Supernova explosions have distributed these nuclei in the Galaxy, hence the relatively large abundance of these nuclei in the solar system.

The origin of the elements beyond iron and nickel, which are not produced by fusion reactions in stars, is still not fully understood. Nevertheless, we do find elements such as iodine, gold, or uranium in nature, so they have to be made somehow. We believe that the vast majority of these elements has been produced by neutron capture processes: a seed nucleus captures a number of neutrons until a radioactive isotope of the same element is formed. When this radioactive isotope decays by beta decay a new, heavier element is created. Successive neutron captures and beta decays can build heavier and heavier elements. Such neutron capture processes tend to form particularly large abundances of nuclei with closed neutron shell configurations, that occur at the "magic" neutron numbers $N=82$ and 126. Similar to the closed electron shells of noble gases in atomic physics, the additional capture of a neutron on nuclei with such magic neutron numbers is hindered by the reduced energy gain. This is a direct consequence of the tiny change in nuclear mass by about 1:100,000 due to the closed neutron shell configuration. The result is a slowdown of the neutron capture process at this point that leads to the build up of particularly large amounts of nuclei. The corresponding peaks in the solar abundance distribution can be found in Fig. 1 at the mass numbers $130,138,195$, and 208. So again it is the nuclear masses and the resulting neutron binding energies that point to the origin of heavy elements by neutron capture. As 
Fig. 1 shows, the abundance maxima occur pair wise. From this one can conclude that heavy elements are formed by two different neutron capture processes - a slow process (s-process) and a rapid process ( $r$-process). The $s$ - and the $r$ - processes cross the closed neutron shells at different elements, so that one obtains two distinct abundance maxima at different mass numbers for each neutron shell closure.

\section{The s-process}

During the s-process neutron captures are in most cases much slower than beta decays. Radioactive isotopes formed after a neutron capture decay quickly into stable nuclei before the next neutron is captured. The s-process proceeds therefore along the so called valley of stability (Fig. 2 ) and crosses the $N=82$ and $N=126$ neutron shells at stable nuclei with mass numbers $A=138$ (barium) and $A=208$ (lead). The abundance maxima created by the s-process at these locations can be readily identified in Fig. 1. From the observation of technetium, which is an element without a stable isotope, on the surface of red giant stars the ongoing operation of the s-process has been demonstrated and the astrophysical site has been identified unambiguously. Because the masses and other properties of the nuclei participating in the s-process are known rather well one can calculate s-process abundances in stellar models quite reliably. Exceptions are the relatively uncertain neutron capture rates of some isotopes, especially in the beginning of the s-process. The experimental determination of precise neutron capture rates for the s-process is an important topic of current research in nuclear astrophysics [4]. Measurements of neutron capture rates on longer lived radioactive isotopes that can do both, capture neutrons and beta decay, so called s-process branching points, represent a particular challenge for future experiments. Important open questions also include the processes in red giants that produce the neutrons needed for the s-process. Poorly understood mixing processes in the stellar interior are particularly relevant, but uncertainties in the neutron producing nuclear reactions are a problem as well [5].

\section{The r-process}

The r-process is responsible for the origin of about half of the heavy elements beyond iron. Elements such as europium, gold, platinum, or uranium are mainly produced in the r-process $[6,7]$. From the location of the abundance maxima in Fig. 1 at $A=130$ (tellurium) and $A=195$ (platinum) one can conclude that the $\mathrm{r}$ process crosses the $N=82$ and $N=126$ neutron shells at nuclei with a mass number around $A=130$ and $A=195$ (see Fig. 2). These nuclei are extremely unstable. ${ }^{130} \mathrm{Cd}(A=130, N=82)$ is a cadmium isotope with a lifetime of only 162 milliseconds, and $195 \mathrm{Tm}(A=195, N=126)$ is an exotic thulium isotope which has never been observed in a laboratory. Obviously the neutron densities during the r-process are so large that neutron captures are much faster than beta decays so that extremely beta unstable nuclei can be formed. After their creation in the r-process these nuclei are converted into stable nuclei through a long chain of beta decays. In some cases these beta decays can emit neutrons, so the mass number of the final stable nucleus tends to be slightly lower than the mass number of the nucleus formed originally in the $r$ process, an effect which we neglected in our simple argument above.

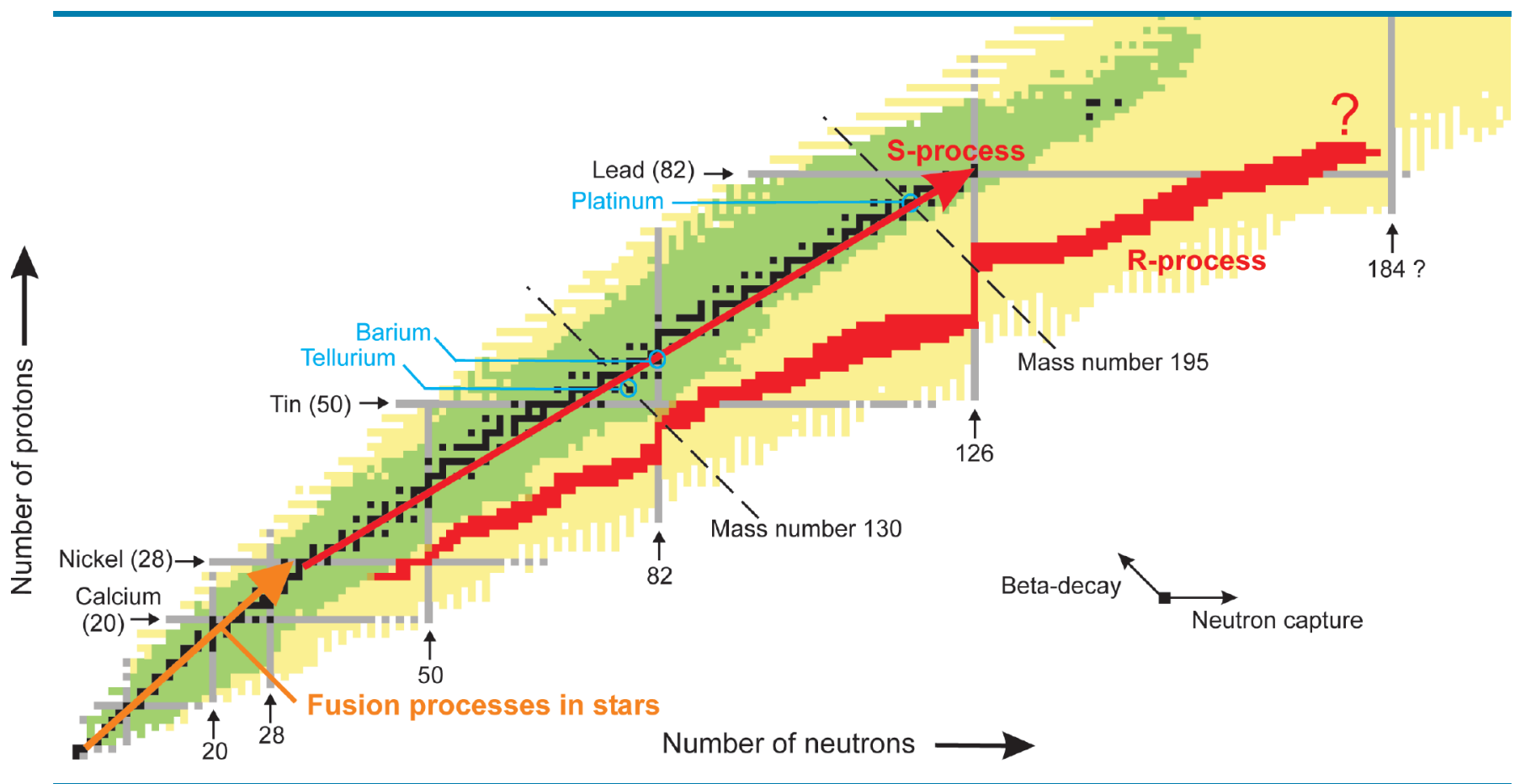

$\Delta$ Fig. 2: Typical predicted path [3] of the r-process (in red) on the chart of nuclides. On this chart, the number of neutrons increases column wise to the right, while the number of protons (element number) increases row wise upward. Each box therefore represents a nucleus with a specific number of protons and neutrons. Nuclei within a horizontal row represent the different isotopes of a given chemical element. Marked in grey are the "magic" proton and neutron numbers, for which the respective shells are closed. Shown are nuclei, which are stable or so long lived that they naturally exist (black), unstable nuclei for which the mass is known (green) and all other unstable nuclei that are predicted by nuclear theory to exist (yellow). The diagonal dashed lines mark the mass numbers where the r-process abundance maxima occur (see Fig. 1). Also shown in a very schematic way is the path of the fusion processes in stars (orange arrow) and the s-process (red arrow). For neutron capture $N$ increases by one, while for a beta decay $Z$ is increased by 1 and $N$ is decreased by 1 (see arrows). 


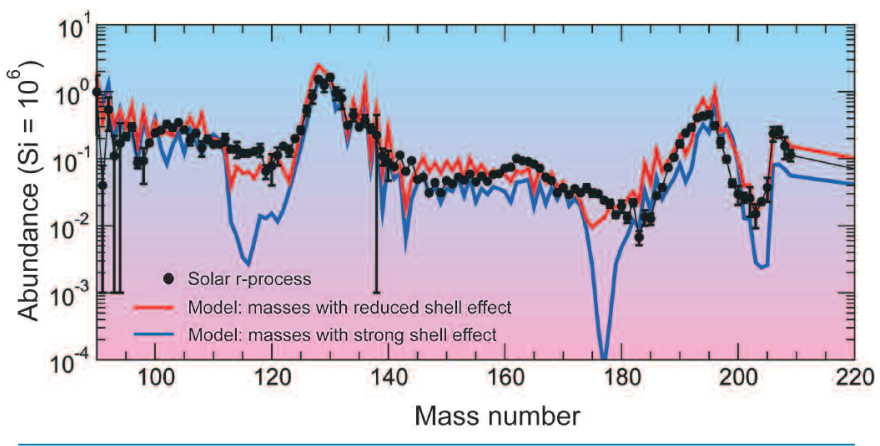

$\Delta$ Fig. 3: Contribution of the r-process to the isotopic abundances of the chemical elements in the solar system as a function of the mass number ("solar r-process"). This distribution is obtained by subtracting calculated contributions from the s-process and other, less important, processes from the observed solar abundance distribution shown in Fig. 1. The abundances are given in usual units relative to one million silicon nuclei. In addition we show the calculated abundances produced in two r-process models that only differ in the theoretical model used to predict the nuclear masses. One mass model shows pronounced shell closures, while the other has a reduced shell closure effect that might occur for very neutron rich nuclei (Data from K.-L. Kratz and B. Pfeiffer, University of Mainz, Germany [7]). This illustrates nicely the strong dependence of predicted r-process abundances on the nuclear masses.

To understand the r-process is one of the greatest challenges of modern nuclear astrophysics. So far it is not known with certainty where in the universe this process can occur. The difficulty for astrophysicists is to find ways to produce the required extreme neutron densities. One possibility is the neutrino flux that drives a wind of material off a hot neutron star forming in the core of a supernova explosion. Though it has been shown that an r-process can occur in such a neutron rich environment, it is still unclear how one can obtain the highest neutron densities needed to produce the heaviest r-process nuclei $[8,9]$. Another possibility is the merging of two neutron stars into a black hole in a neutron star binary system. Model calculations have shown that very neutron rich material can be ejected in such an environment, but simulations also showed that such neutron star mergers might not be frequent enough to explain the observed abundances of heavy elements. Other suggestions for the r-process site include jets in supernova explosions, or gamma-ray bursts from collapsars created by the collapse of a very massive star into a black hole.

New astronomical observations over the last decade have led to dramatic progress in our understanding of the r-process [10]. Of particular importance is the discovery of a few stars in the halo of our Galaxy that are, compared to the sun, extremely iron poor, but strongly enriched in r-process elements. It is believed that these stars are so old that at the time of their formation the composition of the Galaxy was generally iron poor (iron is produced over time by supernova activity) and still extremely inhomogeneous. The r-process element enriched stars apparently formed from interstellar gas that was polluted by a nearby r-process event. In contrast to the solar abundances shown in Fig. 1, which are the product of probably hundreds of different nucleosynthesis events mixed together, the elemental abundances that one can measure through the detection of absorption lines in the photospheres of these stars allow one to determine the products of perhaps a single r-process event. From the few stars found so far one finds for a number of elements good agreement from star to star and also with the r-process contribution to the solar system, though there are interesting discrepancies that might point to the existence of multiple r-processes. In the coming years the discovery of maybe hundreds of such r-process enriched iron poor stars is expected from large scale astronomical survey programs such as HERES (Hamburg/ESO R-process Enhanced Star Survey) or SEGUE (Sloan Extension for Galactic Understanding and Exploration), part of a follow-up program to the Sloan Digital Sky Survey. The results are expected to lead to a new understanding of how neutron capture processes such as the r-process have enriched our Galaxy step by step with heavy elements.

Similar progress is now needed in nuclear physics. Without knowledge of the nuclear physics in the r-process, predictions from theoretical r-process models cannot be compared to observations in a meaningful and quantitative way. The understanding of the r-process is hampered by the fact that hardly any of the extremely unstable participating nuclei could be produced and studied at accelerator laboratories. Nuclear masses play an especially important role for the understanding of the r-process [7]. Most modern r-process models predict that the r-process occurs at high temperatures of a billion degrees or more. At such high temperatures energetic photons can excite nuclei so that they emit neutrons. Such photodisintegration reactions can counteract the rapid neutron captures. At which nucleus this happens within an isotopic chain depends mainly on the binding energy of the neutrons in the nuclei and therefore on the nuclear masses. At the nucleus where photodisintegration wins over neutron capture, the so called r-process waiting point, the r-process will temporarily stop and wait for the beta decay into the next isotopic chain, where the balance of neutron capture and photodisintegration is played out anew. For a given neutron density and temperature nuclear masses must be known in some cases to an accuracy of better than one part in one million to determine the waiting point nuclei. Nuclear masses therefore largely determine the path of the r-process on the chart of nuclides. Together with the beta decay half-lives of the waiting point nuclei, the masses determine also the speed of the process and the final abundance pattern. Precise nuclear masses are therefore essential to compare theoretical abundance patterns with observations. Without reliable nuclear physics the high precision observational data obtained with sophisticated telescopes cannot be quantitatively interpreted.

Unfortunately, most of the extremely neutron rich nuclei in the r-process are still beyond the reach of nuclear physics accelerator facilities. Exceptions

\footnotetext{
>Fig. 4: a) Sketch of a

hyperbolic Penning trap

(diameter about

$2 \mathrm{~cm}$ ). The motion of charged particles inside the trap is a superposition of three

independent eigenmotions: a harmonic oscillation in the axial direction and in radial direction the magnetron and modified cyclotron motion.

b) Total ion motion and projection onto the $x-y$-plane. The motional amplitudes of the ions are less than $1 \mathrm{~mm}$.
}

(a)

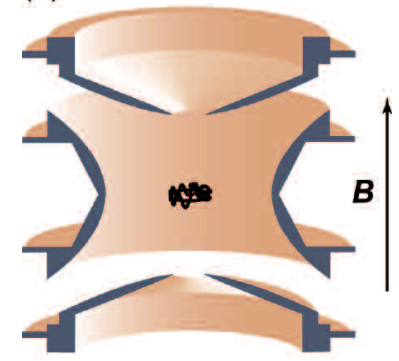

(b)

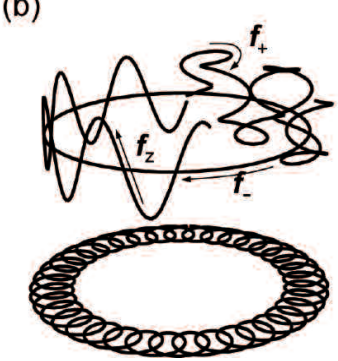


have been pioneering half-life measurements for a few dozen $r$ process nuclei. Such half-life measurements can now be performed with the smallest beam intensities of only a few ions per day $[7,11]$. For the masses of r-process nuclei, on the other hand, one still has to rely on the theoretical predictions of nuclear mass models [12]. These calculations are by far not accurate enough - different models predict masses that differ by as much as $1: 10,000$, about a factor of 100 more than the accuracy needed for r-process calculations. In addition, fundamental changes in the structure of extremely neutron rich nuclei, which are poorly understood because of the lack of experimental data, could lead to even larger systematic errors in mass models. As an example, Fig. 3 shows the predicted abundances from r-process calculations obtained by using two different mass models. One model assumes that the size of the shell gaps and therefore the magnitude of the mass differences associated with closed neutron shells are reduced for extremely neutron rich nuclei. Such a reduction of shell effects is indeed predicted by some nuclear structure models. With such a modified mass model one does obtain a better agreement with observations [13]. However, as long as these possible systematic changes in masses are not confirmed experimentally one cannot decide which calculation uses the correct masses and whether instead fundamental problems in the astrophysical r-process model are to blame for the discrepancies. Less precise methods such as the determination of the electron energy spectrum from beta decay have provided recently a first hint for changes in the shell structure near $N=82$ [14]. However, direct precision mass measurements of extremely neutron rich nuclides are of critical importance. The two most important methods in that respect will be presented in the following sections.

\section{Precision mass measurements far from stability}

Beams of extremely unstable neutron-rich nuclei can already be produced at current accelerators like ISOLDE at CERN in Geneva, Switzerland, at the heavy ion research institute (GSI) in Darmstadt, Germany, or at the NSCL facility at Michigan State University, USA [15]. To this end, a target is bombarded with accelerated stable nuclides. This leads to the production of exotic nuclides by fission or fragmentation, which are then available as ion beams for mass measurements. The challenge is to start the production process with as intensive stable beams as possible, to ionize and transport the exotic nuclei with little losses to the experiment, and to develop highly efficient experimental systems that can perform the measurements with very low beam intensities. The most modern techniques that have been developed at the facilities mentioned above made it already possible to produce nuclides close to or in some cases even in the r-process path with barely sufficient intensities for mass measurements.

The precision of mass determination necessary for $r$-process calculations, is routinely achieved by Penning trap mass spectrometers [15] like ISOLTRAP at ISOLDE/CERN [16] or LEBIT at the Michigan State University [17]. However, these devices only allow to access nuclides with lifetimes of several 10 milliseconds. The masses of short-lived nuclides with lower lifetimes far from stability can only be determined by the time-of-flight method, as e.g. in the experimental storage ring ESR at GSI at Darmstadt [18].

\section{The Penning-trap mass spectrometer}

The Lorentz force of a magnetic field confines the ions inside the Penning trap on a specific orbit. Since there is no force in the direction of the magnetic field lines, i.e. in the axial direction, a three-dimensional confinement is obtained by a superposition of the magnetic field with a weak static electric quadrupole potential.

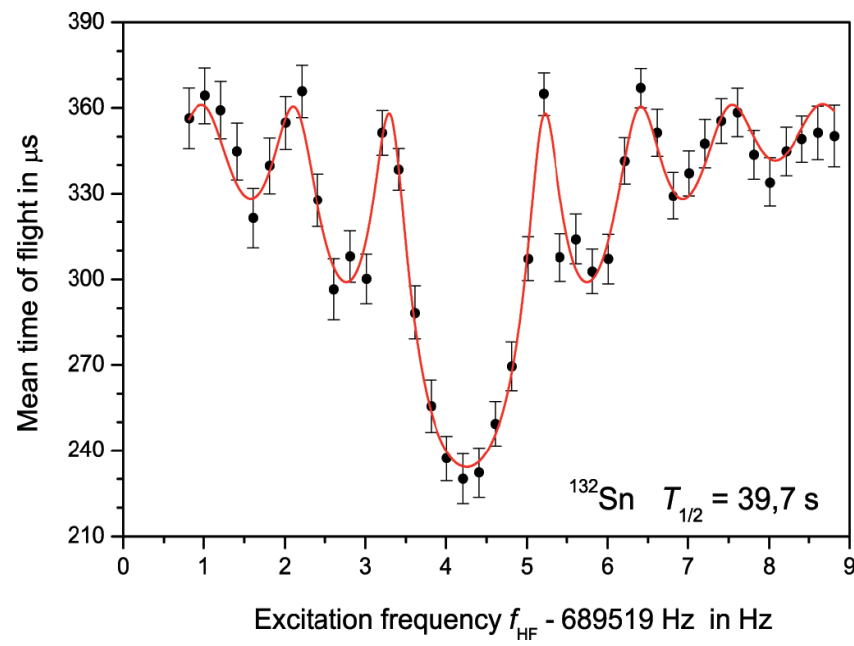

$\Delta$ Fig. 5: Time-of-flight cyclotron resonance curve of the short-lived radionuclide ${ }^{132} \mathrm{~S} n$ with a half-life of $39.7 \mathrm{~s}$. The solid line is a fit of the theoretically expected line shape to the data.

The geometry of a precision Penning trap can be realized by electrodes of hyperbolic shapes, where the surfaces are identical to the equipotential surfaces of the quadrupole potential (Fig. 4a).

The equations of motion for an ion in the resulting potential describe a trajectory consisting of the three independent harmonic oscillations as shown in Fig. $4 \mathrm{~b}$ : the magnetron $\left(f_{-}\right)$, the modified cyclotron $\left(f_{+}\right)$(both radial) and the axial frequency $\left(f_{\mathrm{z}}\right)$ [15]. Note, the sum of the frequencies of the two radial motions, $f_{-}+f_{+}=f_{c}$, is equal to the cyclotron frequency and consequently equal to the revolution frequency of an ion with charge $q$ and mass $m$ in a pure magnetic field $B$ :

$$
f_{\mathrm{c}}=\frac{1}{2 \pi} \cdot \frac{q B}{m}
$$

The cyclotron frequency can be determined by the so called "timeof-flight cyclotron resonance method". Here, the motion of the stored ions inside the Penning trap is first excited by a radiofrequency $f_{\mathrm{HF}}$ close to $f_{\mathrm{c}}$ and subsequently the ions are ejected towards a detector. In case of a resonant excitation the radial energy of the ion increases, resulting in a reduced time of flight to the detector. The reason is that while passing through the strong magnetic field gradient the radial energy of the ion is converted into axial energy. The maximum energy gain and thus the shortest time of flight results for $f_{\mathrm{HF}}=f_{\mathrm{c}}$. Therefore, when measuring the time of flight as a function of the excitation frequency one finds a characteristic resonance (Fig. 5). The centre frequency of the resonance determines the cyclotron frequency and thus according to Eq. (1) the mass of the ion of interest, provided the magnetic field strength is known. To this end, before and after the actual measurement the procedure is also performed with a suitable ion with well-known mass for calibration.

The worldwide best Penning trap mass spectrometers allow one to measure the masses of radionuclides with production rates of only 100 ions per second, with half-lives as short as a few $10 \mathrm{~ms}$ and with an uncertainty as low as $10^{-8}$ [15]. Recently, several nuclides that are relevant for the r-process could be measured with high accuracy at ISOLTRAP. These include masses around the shell closures $N=50$ (e.g.. ${ }^{80} \mathrm{Zn}$ and ${ }^{81} \mathrm{Zn}$ ) and $N=82$ (e.g. ${ }^{132} \mathrm{Sn}$ and $\left.{ }^{133} \mathrm{Sn}\right)$, where one takes advantage of the fact that the r-process gets closer to the stable nuclei (see Fig. 2) [19]. 


\section{Schottky-Mass-Spectrometry}

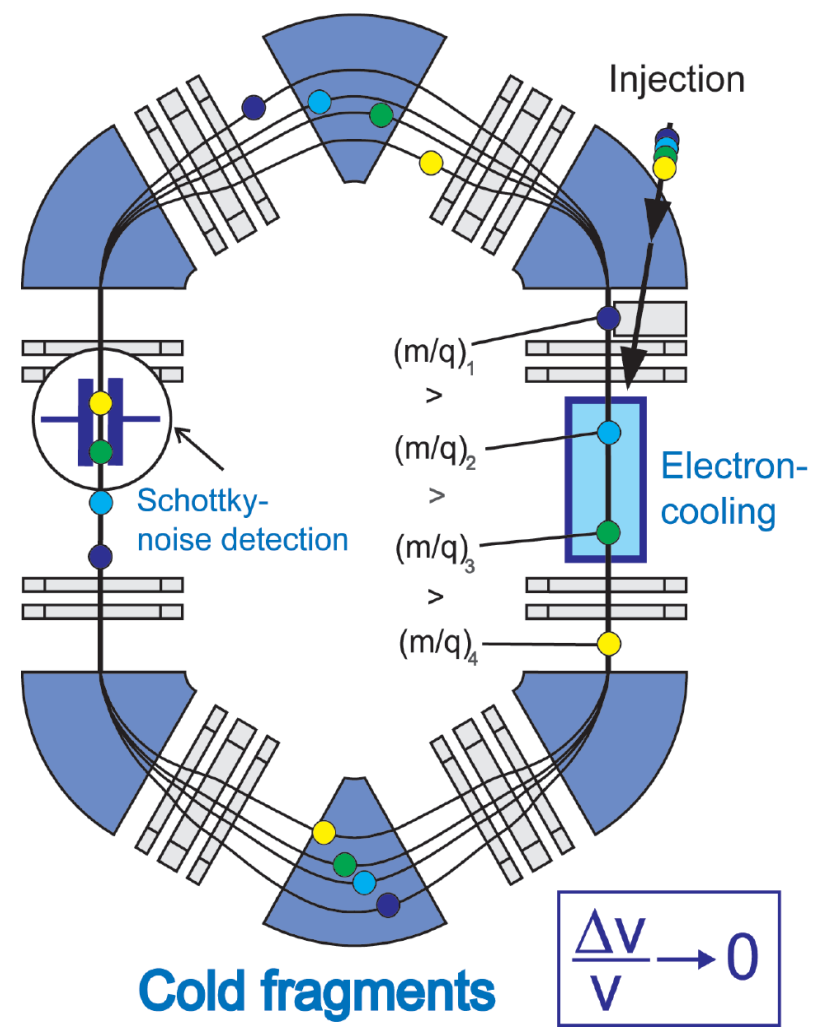

\section{Isochronus-Mass-Spectrometry}

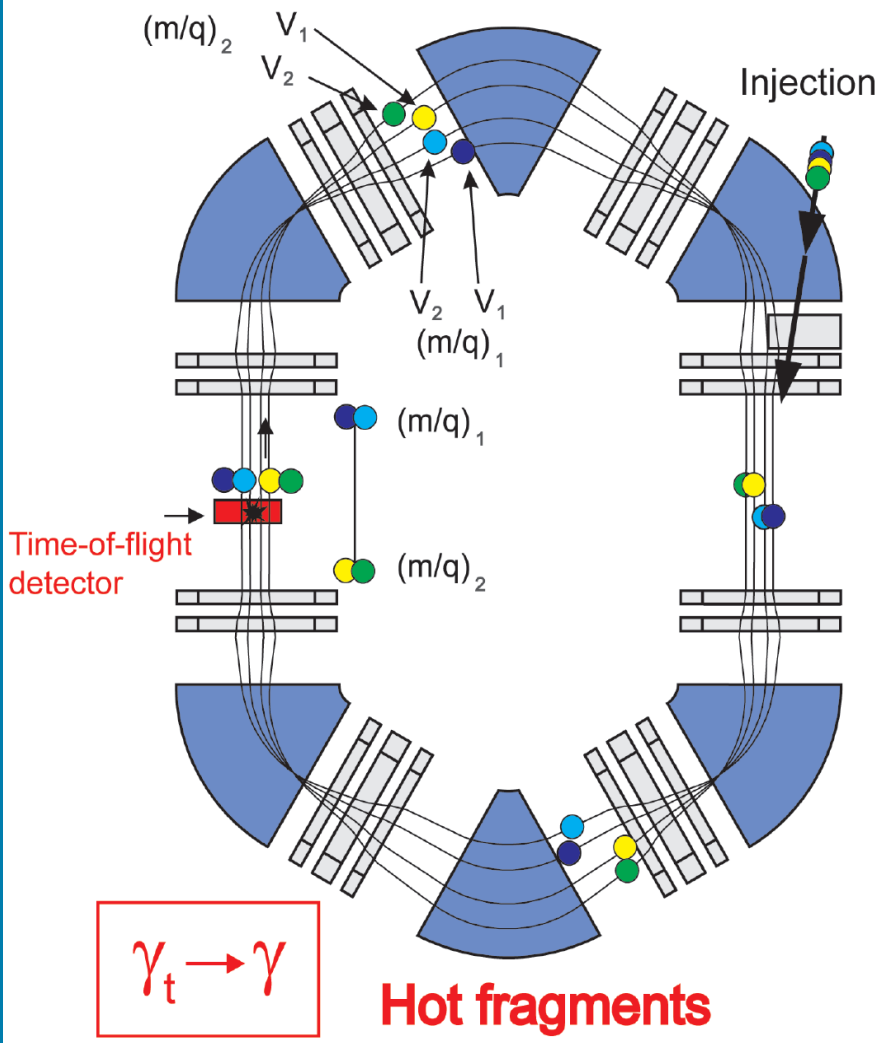

The experimental storage ring

A second, very efficient method for mass measurements on radionuclides is mass spectrometry in a storage ring (Fig. 6). In this case, electric and magnetic fields are used for the three-dimensional storage of the ions and masses are determined from the revolution frequency in the ring. The relation between revolution frequency $f$, mass-to-charge ratio $m / q$ and velocity $v$ of different circulating ions in a storage ring is given by:

$$
\frac{\Delta f}{f}=-\frac{1}{\gamma_{t}^{2}} \frac{\Delta(m / q)}{m / q}+\frac{\Delta v}{v}\left(1-\frac{\gamma^{2}}{\gamma_{t}^{2}}\right)
$$

The quantity $\gamma$ is the Lorentz factor of the ions and $\gamma_{t}$ is an ion-optical parameter of the storage ring. For an unambiguous relation between revolution frequency and mass, the second (velocity dependent) term on the right-hand side of Eq. (2) must be cancelled. Two complementary methods apply to achieve this. In case of the so called Schottky mass spectrometry method (Fig. 6) electron cooling is applied so that $\Delta v / v \rightarrow 0$ [20]. The revolution frequency is measured by a Schottky noise analysis, i.e. at each turn the induced mirror charges of the circulating ions on two electrostatic pick-up electrodes are monitored and amplified. The Fourier transformed signal delivers the frequency and thus the mass spectrum (Fig. 7). The simultaneous storage of known masses for the calibration of the spectrum and unknown masses allows to measure up to several hundred nuclides with an uncertainty of a few $10^{-7}$ in a single experiment. Because the electron cooling takes time, this method can only be applied for nuclides with half-lives of the order of seconds or more. To overcome this limitation, the somewhat less precise method (uncertainties of about $10^{-6}$ ) of isochronous mass spectrometry has been used successfully. In this case the storage ring is operated in an isochronous mode where $\gamma=\gamma_{t}$ [21] such that the orbit frequency of stored ions with the same mass-to-charge ratio is independent of their velocity and the right-hand term in Eq. (2) vanishes. This technique enables a mass determination of radionuclides with lifetimes as low as a millisecond or even less. For the detection of the circulating ions a foil is mounted in the ring aperture and the secondary electrons that are produced at any passage of an ion through the foil are detected. The ions circulate typically a few hundred to a few thousand times in the storage ring with a revolution time of about 0.5 microseconds.

\section{Summary and outlook}

Precision measurements of nuclear masses are of importance for the understanding of nuclear processes in stars and supernova explosions and the origin of the chemical elements in nature. Altogether, the masses of about 1000 short-lived radionuclides have been measured so far using Penning trap and storage ring mass spectrometry $[2,15]$. This huge progress in nuclear physics, which already had impact on astrophysical models, not only required advances in measurement techniques, but also in the production of the exotic unstable nuclei at powerful radioactive ion beam facilities. At ISOLDE at CERN in Geneva extremely

4Fig. 6: Schematic view of the principle of the Schottky and isochronous storage ring measurement method. The circumference of the storage ring is about $110 \mathrm{~m}$. For Schottky mass spectrometry all ions are cooled down to the same mean velocity by electron cooling. Because of their different mass-to-charge ratio $(\mathrm{m} / \mathrm{q})$, the ions perform trajectories with different lengths. In the case of isochronous mass spectrometry no cooling is involved and the ions have different velocities. 
neutron-rich and short-lived tin isotopes close to the r-process path have already been produced and the masses have been measured with unprecedented accuracy in a Penning trap. At GSI in Darmstadt a few nuclides in the $\mathrm{r}$-process path have been produced recently and have been measured using the storage ring method. Meanwhile, a novel method has been developed at the National Superconducting Cyclotron Laboratory at Michigan State University. Exotic nuclides are produced by fragmentation of a heavy nucleus, which is accelerated up to about $30 \%$ of the speed of light. After deceleration in a gas cell the exotic nuclides are injected into a Penning trap. With this method several nuclides in the r-process are within reach for precise mass measurements in the near future. In spite of this enormous progress the majority of nuclides that are important for the stellar nucleosynthesis cannot be produced and research facilities with unprecedented rare isotope production capabilities are planned or under construction, e.g. SPIRAL2 at GANIL, France, FAIR at GSI in Darmstadt, Germany or a new advanced rare isotope accelerator in the US. This new generation of facilities is intended to produce the majority of the nuclides participating in the astrophysical r-process with production rates sufficient for lifetime and mass measurements. Together with the expected progress in astronomy, detailed experimental tests of r-process models will be possible over the next decade and will provide new insights into the origin of the heavy elements in nature.

\section{About the authors}

Hendrik Schatz studied Physics in Karlsruhe and received his Ph.D at Heidelberg. After postdoctoral positions at UC Berkeley and GSI, Darmstadt he joined the faculty at Michigan State University and the National Superconducting Cyclotron Laboratory. $\mathrm{He}$ is now full professor and co-founder of the Joint Institute for Nuclear Astrophysics.

Klaus Blaum studied Physics in Mainz and received his Ph.D from there. After a postdoctoral position at GSI, Darmstadt, he went to CERN to lead the ISOLTRAP experiment. Since 2004 he is group leader of a Helmholtz-Research-Group at the University of Mainz. measured by means of the current facilities. For that reason new

He has already received many awards, among them the GustavHertz-Prize 2004 of the German Physical Society (DPG) and the Mattauch-Herzog-Prize 2005 of the German Society for Mass Spectrometry (DGMS).

\section{References}

[1] E. Anders und N. Grevesse, Geochim. Cosmochim. Acta 53, 197 (1989)

[2] G. Audi, A. H. Wapstra, and C. Thibault, Nucl. Phys. A 729, 3 (2003)

[3] H. Schatz et al., Ap. J. 579, 626 (2002)

[4] F. Kaeppeler, Nucl. Phys. A 752, 500c (2005)

[5] F. Kaeppeler, Prog. Part. Nucl. Phys. 43, 419 (1999)

[6] J. J. Cowan, F.-K. Thielemann, and J.W. Truran, Phys. Rep. 208, 267 (1991)

[7] B. Pfeiffer, K.-L. Kratz, F.-K. Thielemann, and W. B. Walters, Nucl. Phys. A 693, $282(2001)$

[8] S.E. Woosley and R.D. Hoffman, Ap. J. 395, 202 (1992).

[9] K. Takahashi, J. Witti, and H.-Th. Janka, A\&A 286, 857 (1994)

[10] J.W. Truran, J. J. Cowan, C. A. Pilachowski, and C. Sneden, Publ. Astr. Soc. Pac. 114, 1293 (2002)

[11] P. Hosmer et al., Phys. Rev. Lett. 94, 2501 (2005)

[12] D. Lunney, J. M. Pearson, and C. Thibault, Rev. Mod. Phys. 75, 1021 (2003)

[13] B. Chen et al., Phys. Lett. B 355, 37 (1995)

[14] I. Dillmann et al., Phys. Rev. Lett. 91, 2503 (2003)

[15] K. Blaum, Phys. Rep. 425, 1 (2006)

[16] F. Herfurth et al., J. Phys. B 36, 931 (2003)

[17] G. Bollen et al., Nucl. Phys. A 746, 597c (2004)

[18] H.-J. Kluge, K. Blaum, and C. Scheidenberger, Nucl. Instrum. Meth. A 532, 48 (2004)

[19] G. Sikler et al., Nucl. Phys. A 763, 45 (2005)

[20] T. Radon et al., Phys. Rev. Lett. 78, 4701 (1997); T. Radon et al., Nucl. Phys. A 677, 75 (2000)

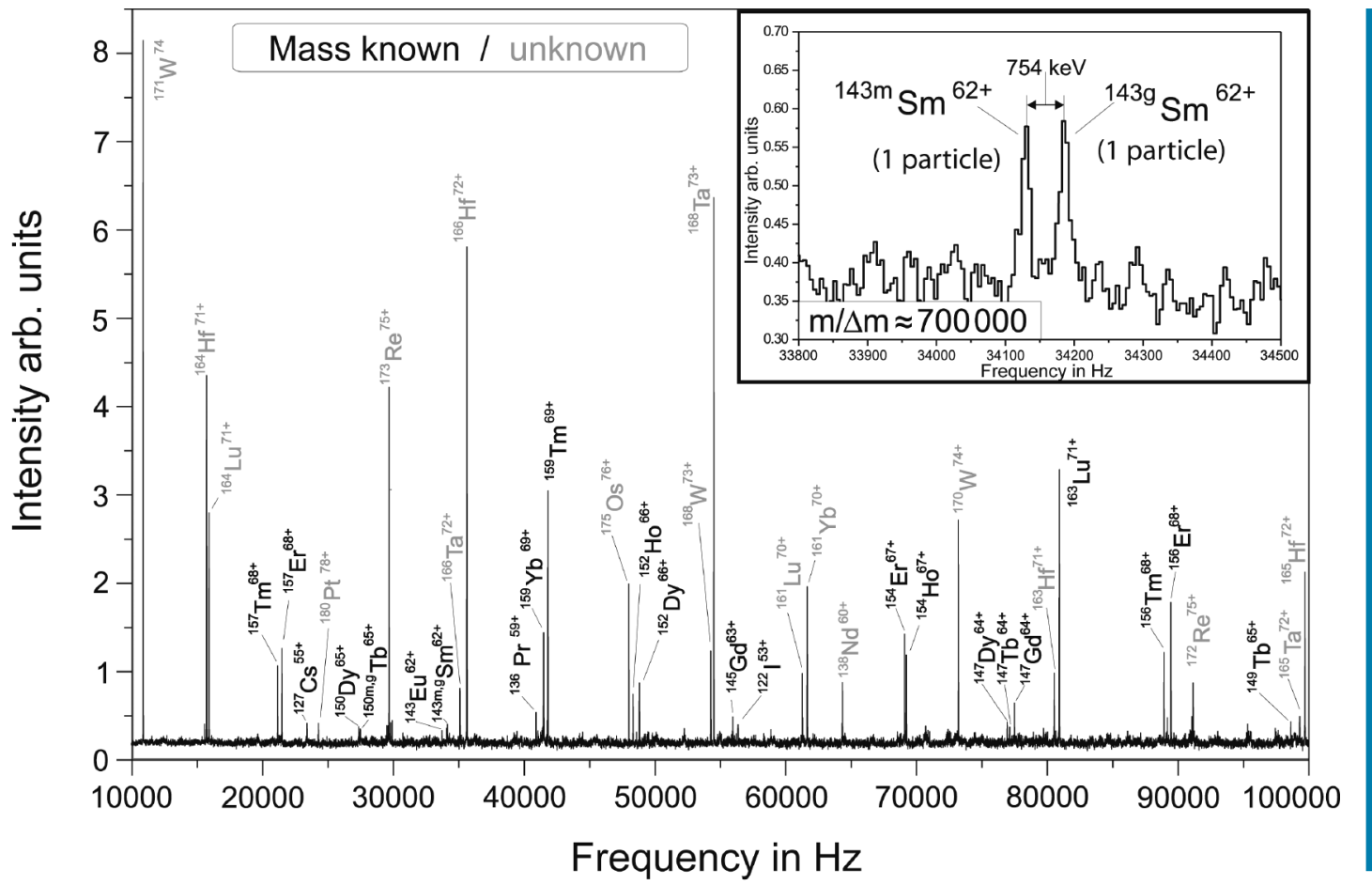

[21] M. Hausmann et al., Nucl. Instr. Meth. A 446, 569 (2000)
Fig. 7: Schottky spectrum of stored exotic nuclides, recorded in the storage ring. The frequency axis shows the difference of the $32^{\text {th }}$ harmonics of the corresponding revolution frequencies of the ions $(\mathrm{v} / \mathrm{c} \approx 0.67)$ with respect to the frequency of a stabilized local oscillator operating at about $59.33 \mathrm{MHz}$. The inset shows ground and isomeric state of fully stripped ${ }^{143} \mathrm{Sm}$. 